\title{
EFEITOS DA OMISSÃO DE NUTRIENTES NOS COMPONENTES DE PRODUTIVIDADE DO ARROZ EM LATOSSOLOS DO NORDESTE PARAENSE
}

\author{
Danielly Cristina da Silva Marques ${ }^{1}$; Juliana Souza da Silva²; José Darlon Nascimento \\ Alves $^{3}$; Morieli Ladislau de Oliveira ${ }^{4}$; Josué Valente Lima ${ }^{1}$; Ricardo Shigueru \\ Okumura ${ }^{5}$.

\begin{abstract}
1Universidade Federal Rural da Amazônia. Capitão Poço, Pará, Brasil. marquesdanielly@ hotmail.com 2Universidade Federal Rural da Amazônia. Capitão Poço, Pará, Brasil. ac_ju@ @otmail.com ${ }^{3}$ Universidade Federal Rural da Amazônia. Capitão Poço, Pará, Brasil. jose.darllon@ @otmail.com ${ }^{4}$ Universidade Federal Rural da Amazônia. Capitão Poço, Pará, Brasil. morieliladislau @ hotmail.com ${ }^{5}$ Universidade Federal Rural da Amazônia. Capitão Poço, Pará, Brasil. ricardo.okumura@ufra.edu.br
\end{abstract}

\begin{abstract}
RESUMO: O presente estudo tem por objetivo avaliar o efeito da omissão de macro e micronutrientes no crescimento de plantas de arroz em Latossolos de textura média e argilosa. $\mathrm{O}$ experimento foi conduzido na casa de vegetação da Universidade Federal Rural da Amazônia (UFRA), no município de Belém-PA. O delineamento experimental utilizado foi o inteiramente casualizado com 11 tratamentos e dois substratos com diferentes texturas (média e argilosa), com três repetições. Os tratamentos avaliados foram: T1) Controle; T2) Completo $($ Calagem + macronutrientes + B, Zn, Cu, Si, Ni); T3) NPK + B, Zn, Cu, Mn + Si (sem calagem); T4) Omissão de N; T5) Omissão de P; T6) Omissão de K; T7) Omissão de Ca; T8) Omissão de Mg; T9) Omissão de B, Zn, Cu, Si e Ni; T10) Omissão de Si; e T11) Omissão de Ni. As variáveis avaliadas foram: massa seca das folhas, massa seca dos colmos e massa seca das panículas. As omissões de $\mathrm{K}, \mathrm{P}$ e $\mathrm{N}$ influenciaram diretamente na produção da cultura do arroz. A deficiência de nitrogênio e potássio afetou a produção da cultura, por restringir o crescimento inicial da planta.
\end{abstract}

PALAVRAS-CHAVE: crescimento, nutrição de plantas, Oryza sativa.

\section{EFFECTS OF OMISSION NUTRIENTS IN COMPONENTS OF RICE PRODUCTIVITY IN NORTHEASTERN PARAENSE LATOSOLS}

\begin{abstract}
The present study aims to evaluate the effect of the omission of macro and micronutrients in the growth of rice plants in sandy loam Oxisols and clayey. The experiment was conducted in a greenhouse at the Federal Rural University of Amazonia (UFRA) in the city of Belem-PA. The experimental design was a completely randomized design with 11 treatments and two substrates with different textures (clayey and silty) with three replications. The treatments were: T1) Control; T2) Complete (Liming + macronutrients + B, Zn, Cu, Si, Ni); T3) NPK + B, Zn, Cu, Mn + Si (without lime); T4) N omission; T5) P omission; T6) K omission; T7) Ca omission; T8) Mg omission; T9) Omission of $\mathrm{B}, \mathrm{Zn}, \mathrm{Cu}, \mathrm{Si}, \mathrm{Ni}$; T10) $\mathrm{Si}$ omission; and T11) Ni omission. The variables evaluated were: leaf dry weight, dry weight of stem and panicle dry mass. The omission of $\mathrm{K}, \mathrm{P}$ and $\mathrm{N}$ directly influenced the production of rice. The deficiency of nitrogen and potassium affect crop production, by restricting the initial growth of the plant.
\end{abstract}

KEYWORDS: growth, Oryza sativa, plant nutrition. 
O arroz (Oryza sativa L.) é uma planta da família das gramíneas, no qual os grãos constituem a base alimentar de muitos países, dessa forma, sugeri-se que seja uma espécie de grande potencial para o combate da fome mundial. No âmbito mundial, o arroz é cultivado nos cinco continentes, tanto em regiões tropicais como temperadas, o que demonstra ser uma cultura extremamente versátil, que se adapta a diferentes condições de solo e clima (MELISSA, 2008).

No Brasil a produção de arroz na safra 2011/2012 foi de $11.559 \mathrm{t} \mathrm{ha}^{-1}$, com produtividade de $4.711 \mathrm{t} \mathrm{ha}^{-1}$ (CONAB, 2012).

A produtividade de grãos depende da interação de vários componentes de produção e do ambiente, mostrando que as correlações entre a produtividade de grãos e seus componentes podem ter valores significativos, positivos ou negativos (FAGERIA et al., 2007).

De acordo com Fornasieri Filho e Fornasieri (2006), o nitrogênio é entre os macronutrientes o segundo mais exigido pela cultura do arroz e o mais exportado como produto colhido.

Nesse sentido, o presente estudo tem por objetivo avaliar o efeito da omissão de macro e micronutrientes no crescimento de plantas de arroz em Latossolos de textura média e argilosa.
O experimento foi conduzido em casa de vegetação da Universidade Federal Rural da Amazônia (UFRA), no município de Belém-PA $\left(01^{\circ} 27^{\prime} 21^{\prime \prime} \mathrm{S} 48^{\circ} 30^{\prime} 14^{\prime \prime}\right.$ O).

O delineamento experimental utilizado foi o inteiramente casualizado com 11 tratamentos, dois substratos com diferentes texturas (média e argilosa) e três repetições, totalizando 66 unidades experimentais. Sendo que cada unidade estava constituída por cinco plantas por vaso.

Foram utilizados substratos de diferentes composições, sendo coletados em locais distintos. O LATOSSOLO AMARELO de textura média foi coletado no município de Capitão Poço - PA e o LATOSSOLO AMARELO de textura argilosa foi retirado do município de Paragominas - PA, na profundidade de 0$20 \mathrm{~cm}$, apresentando as seguintes características químicas e físicas do solo (Tabela 1).

Os tratamentos utilizados no experimento foram: T1) Controle; T2) Completo (Calagem + macronutrientes + B, Zn, Cu, Si, Ni); T3) NPK + B, Zn, Cu, Mn + Si (sem calagem); T4) Omissão de N; T5) Omissão de P; T6) Omissão de K; T7) Omissão de Ca; T8) Omissão de $\mathrm{Mg}$; T9) Omissão de B, Zn, Cu, Si e Ni; T10) Omissão de Si; e T11) Omissão de Ni. 
Tabela 1. Características das análises químicas e físicas do solo da área experimental, provenientes da camada de 0-0,20 m de profundidade, coletadas em LATOSSOLO AMARELO de textura média (Capitão Poço) e LATOSSOLO AMARELO de textura argilosa (Paragominas).

\begin{tabular}{|c|c|c|c|c|c|c|c|c|c|}
\hline Local & $\mathrm{pH}$ & $\mathrm{P}$ & $\mathrm{K}$ & $\mathrm{Ca}$ & $\overline{M g}$ & $\mathrm{Al}$ & $\mathrm{H}+\mathrm{Al}$ & SB & CTC \\
\hline & $\mathrm{H}_{2} \mathrm{O}$ & $-----m$ & $\mathrm{n}^{-3}----$ & --- & & $---\mathrm{cm}$ & $m^{-3}---$ & & $-\ldots$ \\
\hline $\mathrm{CP}$ & 4,2 & 1,37 & 14 & 1,0 & 0,2 & 0,45 & 3,15 & 1,12 & 4,27 \\
\hline $\mathrm{PA}$ & 3,9 & 0,98 & 32 & 0,6 & 0,4 & 1,00 & 4,62 & 1,08 & 5,70 \\
\hline Local & $\mathrm{V}$ & $\mathrm{m}$ & Areia & Silte & Argila & \multirow{2}{*}{\multicolumn{4}{|c|}{ Textura }} \\
\hline & ------ & 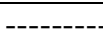 & 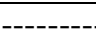 & $\mathrm{g} \mathrm{kg}^{-1}$ & -- & & & & \\
\hline $\mathrm{CP}$ & 26,2 & 28,6 & 733 & 89 & 178 & \multicolumn{4}{|c|}{ Franco arenosa } \\
\hline PA & 18,9 & 48,0 & 76 & 195 & 729 & \multicolumn{4}{|c|}{ Muito argilosa } \\
\hline
\end{tabular}

As fontes e as doses de nutrientes utilizadas no experimento foram: $\mathrm{N}-150$ $\mathrm{mg} \mathrm{kg}{ }^{-1}$ de solo - Uréia; $\mathrm{P}_{2} \mathrm{O}_{5}-200 \mathrm{mg} \mathrm{kg}^{-1}$ de solo - Fosfato de sódio; $\mathrm{K}_{2} \mathrm{O}$ - $100 \mathrm{mg}$ $\mathrm{kg}^{-1}$ - Sulfato de potássio; $\mathrm{Ca}-200 \mathrm{mg} \mathrm{kg}$ ${ }^{1}$ - Cloreto de cálcio; $\mathrm{Mg}-50 \mathrm{mg} \mathrm{kg}{ }^{-1}-$ Cloreto de magnésio; $\mathrm{S}-40 \mathrm{mg} \mathrm{kg}{ }^{-1}-$ Sulfato de sódio; B - $1,0 \mathrm{mg} \mathrm{kg}{ }^{-1}$ - Ácido bórico; $\mathrm{Zn}-5,0 \mathrm{mg} \mathrm{kg}^{-1}$ - Sulfato de zinco; $\mathrm{Cu}-1,0 \mathrm{mg} \mathrm{kg}^{-1}$-Sulfato de cobre; $\mathrm{Si}-200$ $\mathrm{mg} \mathrm{kg}^{-1}$ - Silicato de cálcio e $\mathrm{Ni}-2 \mathrm{mg} \mathrm{kg}-$ ${ }^{1}$ - Cloreto de níquel.

As variáveis avaliadas foram as seguintes massas seca: das folhas, dos colmos e das panículas. Os resultados obtidos foram submetidos ao teste de homocedasticidade e normalidades, em seguida as médias analisados pelo teste de Dunnett $(\mathrm{p}<0,05)$, por meio do software estatístico Assistat.

As massas secas das folhas diferiram significativamente para os tratamentos omissão de $\mathrm{P}$ e controle em relação ao tratamento completo, no solo advindo de
Paragominas (LATOSSOLO AMARELO de textura argilosa), obtendo a menor resposta para esta variável. Enquanto, que no solo de Capitão Poço (LATOSSOLO AMARELO de textura média) verificaramse resultados significativos dos tratamentos omissão de K, P e controle em relação ao tratamento completo, no qual o controle apresentou o menor resultado. Por meio da comparação entre os locais constata-se diferença nos resultados dos tratamentos omissão de $\mathrm{P}$, de Ca e de Mg (Tabela 2).

Em relação à massa seca do colmo, no Latossolo proveniente de Paragominas, apenas o tratamento omissão de $\mathrm{P}$ diferiu estatisticamente do tratamento completo. No solo de textura média, os tratamentos $\mathrm{NPK}+\mathrm{B}, \mathrm{Zn}, \mathrm{Cu}, \mathrm{Mn}+\mathrm{Si}$ (sem calagem), omissão de $\mathrm{N}$, de $\mathrm{K}$ e controle apresentaram resultados diferentes do tratamento completo. Conforme informações apresentadas na Tabela 2, expressiva diferenças são observadas quando compara-se as texturas dos solos 
no tratamento omissão de $\mathrm{P}$, em que a

Paragominas.

menor resposta foi obtida no Latossolo de

Tabela 2. Massa seca da folha e do colmo, de solos provenientes de Latossolo Amarelo de textura argilosa e Latossolo Amarelo de textura média, Estado do Pará.

\begin{tabular}{|c|c|c|c|c|}
\hline \multirow[t]{2}{*}{ TRAT } & \multicolumn{2}{|c|}{ Folha } & \multicolumn{2}{|c|}{ Colmo } \\
\hline & Paragominas & Capitão Poço & Paragominas & Capitão Poço \\
\hline $\mathrm{T} 1$ & $24,62 \mathrm{a}$ & $18,97 \mathrm{a}$ & $6,03 a$ & $6,86^{\mathrm{a}}$ \\
\hline $\mathrm{T} 2$ & n.s. $20,22 a$ & n.s. $24,34 a$ & n.s. $7,87 \mathrm{a}$ & ${ }^{*} 9,52^{\mathrm{a}}$ \\
\hline $\mathrm{T} 3$ & n.s. $13,73 a$ & "8,47a & n.s. $4,77 \mathrm{a}$ & ${ }^{*} 3,53^{\mathrm{a}}$ \\
\hline $\mathrm{T} 4$ & *2,60b & ${ }^{*} 13,45 \mathrm{a}$ & ${ }^{*} 1,09 \mathrm{~b}$ & n.s. $5,05^{\mathrm{a}}$ \\
\hline T5 & n.s. $22,36 a$ & n.s. $14,25 \mathrm{a}$ & n.s. $4,44 a$ & ${ }^{*} 2,58^{\mathrm{a}}$ \\
\hline T6 & n.s. $27,60 \mathrm{a}$ & n.s. $19,21 \mathrm{~b}$ & n.s. $7,38 \mathrm{a}$ & n.s. $6,91^{a}$ \\
\hline $\mathrm{T} 7$ & n.s. $29,58 \mathrm{a}$ & n.s. $17,04 \mathrm{~b}$ & n.s. $5,61 \mathrm{a}$ & n.s. $6,31^{a}$ \\
\hline T8 & n.s. $23,79 a$ & n.s. $18,75 \mathrm{a}$ & n.s. $6,24 \mathrm{a}$ & n.s. $5,79^{a}$ \\
\hline T9 & n.s. $24,84 a$ & n.s. $19,64 a$ & n.s. $5,37 \mathrm{a}$ & n.s. $5,73^{a}$ \\
\hline $\mathrm{T} 10$ & n.s. $25,78 a$ & n.s. $23,30 \mathrm{a}$ & n.s. $6,93 a$ & n.s. $8,42^{a}$ \\
\hline $\mathrm{T} 11$ & ${ }^{*} 5,12 \mathrm{a}$ & *2,45a & ${ }^{*} 1,37 \mathrm{a}$ & ${ }^{*} 1,09^{\mathrm{a}}$ \\
\hline C.V.(\%) & 33,63 & 13,72 & 29,84 & 17,14 \\
\hline D.M.S. & 16,26 & 5,42 & 3,74 & 2,33 \\
\hline
\end{tabular}

"Médias seguidas por um asterisco na mesma coluna diferem significativamente do completo, ao nível de 5\% de probabilidade pelo teste de Dunnett.

n.s. Médias não significativas em relação ao tratamento completo, ao nível de 5\% de probabilidade pelo teste de Dunnett.

${ }^{(1)}$ Médias seguidas de mesma letra minúscula (locais) na linha, não diferem entre si a $5 \%$ de probabilidade pelo teste F.

A pouca disponibilidade de $\mathrm{N}$ e $\mathrm{K}$ influenciaram na produção de massa seca do colmo da cultura do arroz, uma vez que ambos os nutrientes são altamente exigidos pela cultura do arroz de sequeiro (FARINELLI et al., 2004).

A variável massa seca da panícula, no experimento realizado no solo de textura argilosa, apresentou para os tratamentos omissão de $\mathrm{P}$ e controle diferenças estatísticas sobre o tratamento completo. No solo de textura média não ocorreu diferença entre os tratamentos. Comparativamente entre os locais, verifica-se que ocorreram resultados diferentes nos tratamentos omissão de $\mathrm{P}$ e de Ni (Tabela 3).

Para a massa seca da folha bandeira, no LATOSSOLO AMARELO de textura argilosa, houve efeito significativo apenas no tratamento omissão de $\mathrm{Ni}$ em relação ao tratamento completo. Enquanto, em Capitão Poço obteve-se diferença entre as médias dos tratamentos omissão de $\mathrm{K}$ e controle quando comparado ao tratamento completo. Por meio da análise entre os locais, observa uma diferença expressiva nos tratamentos completo, NPK $+\mathrm{B}, \mathrm{Zn}$, 
$\mathrm{Cu}, \mathrm{Mn}+\mathrm{Si}$ (sem calagem), omissão de $\mathrm{P}$, de $\mathrm{Ca}$, de $\mathrm{Mg}$ e de $\mathrm{Si}$, nos quais os menores valores foram obtidos no Latossolo de textura argilosa.

A disponibilidade de $\mathrm{P}$ e $\mathrm{K}$ é essencial para o aumento da produção de massa seca da panícula e da folha bandeira, uma vez que elevadas doses de nitrogênio na cultura do arroz incrementam o número de panículas $\mathrm{m}^{-2}$, número de grãos panícula ${ }^{-1}$ (FARINELLI et al., 2004). Além disso, a aplicação correta de $\mathrm{N}$ na fase reprodutiva contribui para a formação de perfilhos e, portanto, de panículas, enquanto na fase reprodutiva, ocorre a definição do número de grãos por panículas e do peso dos grãos (MARZARI et al., 2005).

Tabela 3. Massa seca da panícula (PAN) e folha bandeira (FB) em solos provenientes de Latossolo Amarelo de textura argilosa e Latossolo Amarelo de textura média, Estado do Pará.

\begin{tabular}{|c|c|c|c|c|}
\hline \multirow[t]{2}{*}{ TRAT } & \multicolumn{2}{|c|}{ Panícula } & \multicolumn{2}{|c|}{ Folha bandeira } \\
\hline & Paragominas & Capitão Poço & Paragominas & Capitão Poço \\
\hline $\mathrm{T} 1$ & $15,86 a$ & $11,35 \mathrm{a}$ & $1,84 \mathrm{~b}$ & $3,35^{\mathrm{a}}$ \\
\hline $\mathrm{T} 2$ & n.s. $10,59 a$ & n.s. $13,16 a$ & n.s. $1,71 b$ & n.s. $2,77^{a}$ \\
\hline $\mathrm{T} 3$ & n.s. $15,14 \mathrm{a}$ & n.s. $10,99 a$ & n.s. $2,56 a$ & n.s. $2,52^{a}$ \\
\hline $\mathrm{T} 4$ & $* 1,60 b$ & n.s. $10,19 a$ & n.s. $1,13 b$ & n.s. $3,63^{a}$ \\
\hline T5 & n.s. $9,34 a$ & n.s. $5,17 \mathrm{a}$ & n.s. $1,94 a$ & ${ }^{*} 1,63^{\mathrm{a}}$ \\
\hline T6 & n.s. $9,58 \mathrm{a}$ & n.s. $12,95 \mathrm{a}$ & n.s. $1,51 \mathrm{~b}$ & n.s. $2,94^{a}$ \\
\hline $\mathrm{T} 7$ & n.s. $8,11 \mathrm{a}$ & n.s. $7,23 a$ & n.s. $2,07 b$ & n.s. $3,12^{\mathrm{a}}$ \\
\hline $\mathrm{T} 8$ & n.s. $12,23 \mathrm{a}$ & n.s. $13,96 a$ & n.s. $2,39 a$ & n.s. $1,89^{\mathrm{a}}$ \\
\hline T9 & n.s. $11,78 \mathrm{a}$ & n.s. $7,14 a$ & n.s. $2,20 b$ & n.s. $3,33^{\mathrm{a}}$ \\
\hline $\mathrm{T} 10$ & n.s. $8,33 b$ & n.s. $17,09 a$ & $* 3,10 \mathrm{a}$ & n.s. $2,55^{\mathrm{a}}$ \\
\hline $\mathrm{T} 11$ & ${ }^{*} 1,62 \mathrm{a}$ & n.s. $2,25 \mathrm{a}$ & n.s. $1,36 a$ & ${ }^{*} 1,03^{\mathrm{a}}$ \\
\hline C.V. $(\%)$ & 46,88 & 56,97 & 21,56 & 23,19 \\
\hline D.M.S. & 10,73 & 13,95 & 1,03 & 1,47 \\
\hline
\end{tabular}

"Médias seguidas por um asterisco na mesma coluna diferem significativamente do completo, ao nível de 5\% de probabilidade pelo teste de Dunnett.

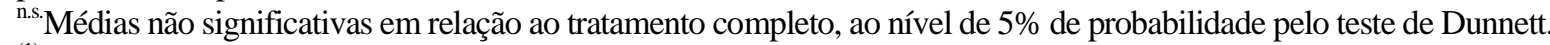

${ }^{(1)}$ Médias seguidas de mesma letra minúscula (locais) na linha, não diferem entre si a $5 \%$ de probabilidade pelo teste $\mathrm{F}$.

A omissão de fósforo na cultura do arroz influencia diretamente o seu crescimento, independente do LATOSSOLO AMARELO ser caracterizado como textura média ou argilosa. A deficiência de nitrogênio e potássio afeta na produção da cultura, por restringir o crescimento inicial da planta.

\section{REFERÊNCIAS}

COMPANHIA NACIONAL DE ABASTECIMENTO. Acompanhamento da safra brasileira: grãos, Safras 2011/2012: décimo levantamento. Brasília: Conab, 2012. Disponível em:<http://www.conab.gov.br/safras.asp.> Acesso em: 12 mai. 2013. 
FAGERIA, N. K. et al. Produtividade de arroz irrigado e eficiência de uso do nitrogênio influenciadas pela fertilização nitrogenada. Pesquisa Agropecuária Brasileira, v. 42, n. 7, p. 1029-1034, 2007. FARINELLI, R. et al. Características agronômicas de arroz de terras altas sob plantio direto e adubação nitrogenada e potássica. Revista Brasileira de Ciência do Solo, v. 28, p. 447-454, 2004.

FORNASIERI FILHO, D.; FORNASIERI, J.L. Manual da cultura do arroz. Jaboticabal: FUNEP, 2006. 589p.

MARZARI, V. et al. Épocas de aplicação do fertilizante nitrogenado no sistema convencional de semeadura de arroz irrigado. Ciência Rural, v. 35 n. 5, p. 1190-1193, 2005.

MELISSA, W. Arroz: composição e características nutricionais. Ciência Rural, v. 38, n. 4, p. 1184-1192, 2008. 